\title{
A98-25238
}

AIAA-98-1988

\section{A NUMERICAL AND EXPERIMENTAL STUDY OF THE RESPONSE OF SELECTED COMPRESSION-LOADED COMPOSITE SHELLS WITH CUTOUTS}

\author{
Mark W. Hilburger * \\ University of Michigan \\ Ann Arbor, MI 48109-2118 \\ James H. Starnes Jr. ${ }^{\dagger}$ \\ NASA Langley Research Center \\ Hampton, VA 23681-0001 \\ Anthony M. Waas $\ddagger$ \\ University of Michigan \\ Ann Arbor, MI 48109-2118
}

\begin{abstract}
$\underline{\text { Abstract }}$
A summary of results from a numerical and experimental study of the response of compression-loaded laboratory scale composite cylindrical shells with rectangular cutouts is presented. The numerical results are obtained using a geometrically nonlinear finite element analysis code. The effects of varying cutout size and laminate properties on the response of compressionloaded shells are described. A detailed finite element model of a selected test specimen is used to provide a more accurate representation of the actual experiment. The detailed model includes measured initial geometric imperfections, nonuniform load introduction, boundary condition nonuniformities, shell thickness variations, and material property variations. Results indicate that the nonlinear interaction between the out-ofplane deformations and in-plane stress resultants near the cutout can significantly influence the structural response of the shell. The results also indicate that these local displacements and internal load distributions can be affected by the size of the cutout and the material properties of the shell.
\end{abstract}

\section{$\underline{\text { Introduction }}$}

Thin-walled cylindrical shells are found in many aerospace structural applications. With the increasing need to produce lighter, stronger, more efficient aerospace structures, the use of advanced composite materials has become more common in the design of

*Ph.D. Candidate, Composite Structures Laboratory, Department of Aerospace Engineering. Student Member, AIAA.

†Head, Structural Mechanics Branch. Fellow, AIAA.

$\ddagger$ Associate Professor, Composite Structures Laboratory, Dept. of Aerospace Engineering. Member AIAA. Copyright (C)1998 by Mark W. Hilburger. Published by the American Institute of Aeronautics and Astronautics, Inc. with permission. these structures. The high strength-to-weight and high stiffness-to-weight ratios of advanced composite materials offer significant weight reduction potential for these type of structures. Many aerospace shell structures have cutouts or openings in the structure to serve as doors, windows, or access ports. Insight into the response characteristics of composite shells with cutouts subjected to typical flight loads and the causes of material failures must be understood and accurately predicted in order to determine safe operating conditions and effective designs for these structures.

A cutout in a shell structure causes a local response to occur in the neighborhood of the cutout when the shell is subjected to load. This local response can consist of large out-of-plane deformations and rapidly varying stress gradients near the cutout (e.g., Ref 1,2 ). If the load is a compressive load, the cutout may also cause a local buckling response to occur at applied loads lower than the general instability load of a shell without a cutout (e.g., Ref 3-5). In some cases this local response results in a stable local postbuckling mode in the shell near the cutout, and additional load can be applied to the shell before it finally buckles into the general instability collapse mode. In other cases the unstable local response causes a local disturbance of sufficient magnitude in the shell to cause the general instability to occur immediately after the local instability occurs. Both responses are accompanied by a significant reduction in the shell stiffness and load carrying capability.

The present paper describes results from a detailed numerical and experimental investigation of the compression response of graphite-epoxy cylindrical shells with rectangular cutouts. Numerical results of a representative shell model are presented first to identify typical response characteristics of a compression-loaded shell with a cutout. These results are followed by a 
comparison of numerically predicted and experimentally measured results for six laboratory scale shells. Two laminate stacking sequences and three cutout sizes were studied to identify the effects of laminate properties and cutout size on the compression response. Finally, results from a detailed finite element analysis of one of the tested shells is presented. The detailed nonlinear analysis includes the effects of initial measured geometric imperfections, nonuniform load introduction, boundary condition nonuniformities, shell wall thickness variations, and material property variations. The investigation presented here is documented in detail in Ref. 6.

\section{$\underline{\text { Experiments }}$}

\section{Test Specimens}

Six eight-ply laminated shells were fabricated on an aluminum mandrel using 12.0-in wide unidirectional Hercules, Inc. AS4/3502 pre-impregnated graphiteepoxy tape. The shells had a nominal radius of 8.0 inches and a length of 16.0 inches. The specimens have $[\mp 45 / 0 / 90]_{s}$ and $\left[\mp 45 / 0_{2}\right]_{s}$ stacking sequences with 0 deg. plies aligned in the axial direction of the shell. The nominal lamina ply thickness is 0.005 inches resulting in a total wall thickness of 0.04 inches. The lamina material properties are summarized in Table 1. After the shells were cured according to manufacturer's recommended procedures, they were ultrasonically inspected to insure initial quality of the shell. A C-scan technique was employed for this task using a pulse-echo method. No significant defects were identified in the shells. A rectangular cutout was machined in each of the cylinders at $\theta=0^{\circ}$, at the shell midlength. The cutouts considered in the present study include a 1.0 -in by 1.0 -in square, a 1.0 -in by 2.0 -in circumferentially aligned rectangle, and a 2.0 -in by $1.0-$ in axially aligned rectangle. Each of the cutouts had re-entrant corners with a 0.05 inch radius. The axial and circumferential cutout dimensions are denoted as $a$ and $b$, respectively. Test specimen identification codes, laminate stacking sequences, and cutout dimensions are summarized in Table 2. To insure that the ends of the specimen remained circular during testing and did not fail prematurely, the ends were potted in an aluminum-epoxy casting material, HYSOL TE-5467 aluminum filled epoxy with HD-0111 hardener. The potting extended approximately 1.0 inch in from both ends of the specimen resulting in a total unsupported shell length of 14.0 inches. The ends of each specimen were then machined flat and parallel. A finished uninstrumented specimen is shown in Figure 1. A schematic of the shell geometry, kinematics, and analytical boundary conditions is shown in Figure 2a.

Three sets of initial imperfections were measured in order to characterize the geometry of each specimen. The measurements included the results of a three-dimensional survey of the inner and outer shell surfaces to characterize the initial mid-surface geometric imperfections, thickness variations, and material property variations in the cylinder. The surface measurements were taken every 0.125 inches along the unsupported length of the specimen and every degree around the circumference. Measurements of the loading surface geometry were obtained to characterize nonuniform load introduction into the specimen, and measurements of the potting thickness were taken in order to characterize any nonuniformities in the boundary conditions.

\section{Instrumentation, Apparatus, and Procedure}

Surface strain measurements were made with backto-back electrical resistance strain gage pairs mounted on the inner and outer surfaces of each specimen. Strain measurements were taken at over 80 locations on each specimen with the majority of the gages positioned near the cutout. Displacements of the test frame loading platens were measured with direct current differential transformers (DCDT's) at three locations on the top loading platen as illustrated in Figure 2b. All electrical signals from the strain gages, DCDT's, and load output were recorded on a magnetic tape data acquisition system, MODCOMP, at 1-second intervals during the test.

The specimens were tested in quasi-static compression using a 300 kip. capacity Southwark Emery hydraulic loading frame. Before each test, a load balancing procedure was used to insure that the loading platens would apply the load to the specimen as uniformly as possible. The load was applied to the specimen via a controlled axial end-shortening displacement. All specimens were loaded to the point of global collapse.

\section{Cylinder Models and Analysis Methods}

The STAGS (STructural Analysis of General Shells) finite element analysis code ${ }^{7}$ was used to predict the compression response of laminated composite shells with cutouts. This code was chosen due to its extensive nonlinear analysis capabilities and its ability to include a variety of imperfections in the model. This section will first present the model and boundary conditions for geometrically perfect shells with a rectangular cutout used in the study. This will be followed by a description of the nonlinear analysis methods used in the solution process. Finally, a discussion of refinements made to the geometrically perfect model is presented. 


\section{Geometrically Perfect Model}

A typical finite element mesh of a shell specimen with a cutout is shown in Figure 2a. The shells were modeled using STAGS 410 quadrilateral elements and 510 and 710 transition elements when necessary. The elements are flat facet type elements based on KirchoffLove shell theory and the nonlinear Lagrangian strain tensor. Each of the element nodes includes six degrees of freedom, three translational degrees of freedom $u, v$, and $w$, and three rotations about the $x, \theta$, and $z$ axis. Large rotations are accounted for using a corotational algorithm. The nodes and elements of the refined mesh near the cutout were defined using user-defined subroutines compatible with the STAGS program. The compression load was introduced into the cylinder by means of a uniform axial displacement. An idealized version of experimental boundary conditions were included in the finite element model by constraining the circumferential and radial degrees of freedom $v$ and $w$, respectively, in regions of the cylinder 0.0 -in $\leq x \leq 1.0$ in and 15.0-in $\leq x \leq 16.0$-in as illustrated in Figure 2a resulting in a total unsupported length of 14.0 inches.

The quasi-static compression response of the shells was determined using a standard arc-length projection method $^{8}$ in STAGS. In many cases, the arc-length projection method was sufficient in obtaining results beyond instability points. However, in cases were the standard arc-length method failed to converge to solutions beyond an instability point, a nonlinear transient analysis method $^{9}$ was used. The transient analysis was initiated at an unstable equilibrium state just beyond the instability point by adding an increment in the end-shortening displacement. The transient analysis continued until the kinetic energy in the system became negligible. A load relaxation procedure was applied to the system to establish a stable equilibrium state from which the quasi-static arc-length method could be restarted. The model identification codes, laminate stacking sequences, and cutout dimensions are summarized in Table 2.

\section{Refined Model}

Refinements in the idealized shell model were made in an effort to produce an accurate model of shell specimen $\mathrm{C} 1$ and are presented herein. The refinements include modeling of initial geometric imperfections, nonuniform load introduction, boundary condition nonuniformities, shell thickness variations, and material property variations. A summary of average adjusted specimen dimensions based on the measured imperfections is given in Table 3.

The measured initial geometric mid-surface imperfection for specimen $\mathrm{C} 1$ normalized by the nominal wall thickness of the shell is shown in Figure 3a. The mid- surface imperfection was calculated by taking an average of inner and outer surface measurements. The imperfection indicates that the specimen was elliptical in shape with small deviations along the axis. The periodic variation of the imperfection around the circumference has a magnitude of approximately four shell wall thicknesses. The perfectly circular shell model was modified to include the mid-surface imperfection by introducing an initial out-of-plane geometric imperfection at each node.

The measured shell wall thickness variation normalized with respect to the nominal wall thickness is shown in Figure $3 \mathrm{~b}$. The imperfection contour indicates that there is a significant variation in the thickness of the specimen. The cross-hatch pattern of the imperfection is due to outer-surface roughness that is caused by a release cloth used during specimen fabrication and ply lap-seams and gaps generated during the layup and curing process. The thinner areas of the specimen are attributed to excess resin flow during the curing process. From the thickness imperfection data, thickness adjusted lamina properties were established using a modified rule of mixtures. ${ }^{6}$ A summary of average thickness adjusted lamina properties is given in Table 4. The uniform thickness and uniform material properties from the perfect shell model were modified to include both the measured thickness imperfections and the adjusted lamina properties at the element level.

Nonuniform load introduction in the specimen is attributed to initial specimen loading surface imperfections and loading platen rotations that occurred during the experiment. The measured loading surface imperfections for specimen $\mathrm{C} 1$ are shown in Figure 3c. The loading surface imperfection was included in the refined model by adding an initial in-plane axial imperfection to the nodes at each end of the shell. This loading surface imperfection results in alternating regions of contact and no contact between the specimen loading surface and the test frame loading platens. In order to model accurately this nonlinear contact problem, the loading platens were modeled as rigid flat plates with generalized contact definitions defined between the flat plates and the shell loading surfaces. To model accurately the applied displacements in specimen $\mathrm{C} 1$, the actual experimentally measured displacements of the loading platens are applied to the top flat plate in the STAGS model while holding the bottom plate fixed. The experimentally measured axial load versus end-shortening displacement and axial load versus endrotations for specimen $\mathrm{C} 1$ are given in Figure $4 \mathrm{a}$ and Figure $4 \mathrm{~b}$, respectively. The results are normalized with respect to classical buckling values for a shell without a cutout subjected to axial compression and bending where appropriate. The results in Figure $4 \mathrm{~b}$ indicate 
that significant rotation of the loading platen occurred during the experiment.

The measured potting thickness is given in Figure $3 \mathrm{~d}$. The data indicate that a variation of approximately \pm 0.012 inches in the thickness of the potting exists and is 0.135 inches thinner than the specified design thickness of 1.0 inch on average. The potting thickness variation is applied to the refined model by adding an initial in-plane axial imperfection to the nodes associated with the idealized boundary conditions illustrated in Figure $2 a$.

\section{$\underline{\text { Results and Discussion }}$}

A summary of the results from a numerical and experimental study of the compression response of composite shells with cutouts is presented. First, numerically predicted results from a representative shell will be presented to identify typical response characteristics of a compression-loaded laminated shell with a cutout. These results are followed by a comparison between predicted and measured responses of six compression-loaded composite specimens with cutouts identifying the effects of cutout size and laminate properties on the response. Finally, a comparison between the measured results of one selected test specimen and a refined numerical model of the specimen is presented.

\section{Predicted Response of a Shell with a Cutout}

The predicted response of the orthotropic $\left[\mp 45 / 0_{2}\right]_{s}$ cylinder $\mathrm{C} 1$ is presented to highlight typical response characteristics of a compression-loaded composite shell with a cutout. The predicted load versus endshortening response curve is given in Figure $5 \mathrm{a}$ as an overall guide to the shell response. The response curve indicates that an extensive postbuckling response is present in a compression-loaded shell with a cutout. The first buckling point is labeled as point $A$ and the additional extreme points are labeled B, C, D, etc. The postbuckling response includes both stable segments (e.g., B-C, D-E) and unstable segments (e.g., A-B, C-D) as shown in the figure. Segment A-B is associated with a local buckling event resulting in large out-of-plane displacements and rapidly varying stress gradients near the cutout. Additional loading in the postbuckling range along segment $\mathrm{B}-\mathrm{C}$ is accompanied by significant changes in the local displacements and stresses in the shell near the cutout leading to the global collapse point of the shell at point $\mathrm{C}$. The collapse response is represented by segment $\mathrm{C}-\mathrm{D}$ on the response curve and was simulated using the transient analysis in STAGS. The collapse response is characterized by a significant reduction in load and the development of the general instability mode in the shell. The load ver- sus time history of this dynamic collapse response is given in Figure 5b. The slope of each adjacent stable equilibrium segment of the response curve decreases as loading continues in the postbuckled range, indicating a reduction in effective axial compression stiffness of the cylinder. This is due to increasingly large deformations that develop in the shell throughout the compression response.

The displacement responses corresponding to the first local buckling mode at point $\mathrm{B}$, the postbuckling mode shape at point $C$, and the global collapse mode shape at point D in Figure 5a are shown in Figure 6a, $6 \mathrm{~b}$, and $6 \mathrm{c}$, respectively. The mode shape associated with point B consists of large elliptical shaped buckles on either side of the cutout with the semi-major axis of the buckles aligned with the axis of the shell. The postbuckling pattern associated with point $\mathrm{C}$ in Figure $6 \mathrm{~b}$ indicates that the elliptical buckles have rotated around the cutout and have become increasingly defined. The displacements are distributed farther around the circumference of the shell leading to a reduction in the effective axial stiffness as indicated in the loadshortening response curve. The displacement pattern shown in Figure 6c is associated with the general instability collapse mode of the shell. The mode shape consists of eight circumferentially distributed diamondshaped buckles. The postcollapse response indicates that the effective axial stiffness and the overall load carrying capacity of the shell is reduced after collapse.

Contour plots of axial and circumferential (hoop) stress resultants corresponding to the first local buckling point at point $\mathrm{A}$ in Figure $5 \mathrm{a}$ are shown in Figure $7 \mathrm{a}$ and $7 \mathrm{~b}$. The results indicate that rapidly varying stress gradients form near the cutout and quickly decay to far-field values away from the cutout. The results also indicate that regions of local biaxial compression form in the shell near the cutout. These regions of destabilizing biaxial stress couple with the out-of-plane deformations near the cutout causing the local buckling response.

\section{Predicted and Measured Response Comparison}

The predicted and measured response of specimens C1 through C6 are presented to identify the effects of cutout size and laminate properties on the compression response. Axial load versus end-shortening relations are presented for these cases as an overall guide to the response of these shells. The axial loads and end-shortening displacements are normalized with respect to the classical buckling values of a shell without a cutout, and the first local buckling points and general instability points are marked with open symbols on the curves.

The predicted and measured axial load versus 
end-shortening response curves for the orthotropic $\left[\mp 45 / 0_{2}\right]_{s}$ specimens C1-C3 are compared in Figures $8 \mathrm{a}$ and $8 \mathrm{~b}$, respectively. The results indicate that $\mathrm{a}$ variation in the size of the cutout can have a significant effect on the compression response of the shell. The results indicate that an increase in the size of the cutout leads to a reduction in the value of first local buckling load and the magnitude of the load drop associated with the ensuing buckling response in the shell. The results indicate that the reduction in the buckling load and the postbuckling load drop is more severe for a circumferentially aligned rectangular cutout than an axially aligned rectangular cutout. The analytical models predict normalized buckling loads of $0.729,0.673$, and 0.606 for specimens $\mathrm{C} 1, \mathrm{C} 2$, and $\mathrm{C} 3$, respectively, and the measured buckling loads are $0.573,0.538$, and 0.515 , respectively. The increase in the size of the cutout in specimens $\mathrm{C} 2$ and $\mathrm{C} 3$ produces larger prebuckling deformations which causes extensive stress redistribution around the circumference of the shell. This stress redistribution causes an overall weakening of the shell at lower applied loads as indicated by the reduction in the buckling loads. The measured and predicted results agree well in this regard. However, the buckling load predictions from the idealized shell models are $19.0 \%$ higher on average than the measured experimental values. This discrepancy is likely to be due to imperfections in the specimens similar to those presented earlier.

The results indicate the first local buckling event can either be an unstable sudden buckling response associated with a sharp reduction in load immediately past the buckling point as predicted for specimens $\mathrm{C} 1$ and $\mathrm{C} 2$, or a gradual buckling response as predicted for specimen $\mathrm{C} 3$ as shown in Figure 8a. In either case, the buckling response results in the formation of large outof-plane deformations and rapidly varying stress gradients near the cutout similar to those predicted for specimen $\mathrm{C} 1$ and shown in Figures $6 \mathrm{a}$ and $7 \mathrm{a}$, respectively. The overall character of the local buckling displacements observed for specimen $\mathrm{C} 1$ through C3 during the experiments agreed well with the predicted displacements.

The predicted postbuckling responses indicate that additional load can be applied to the shell before it buckles into the general instability mode, however, the effective axial compression stiffness is reduced due to large out-of-plane deformations that form in the shell. The measured postbuckling responses indicate that a significantly larger amount of applied end shortening was required to bring about the global collapse response in the shells as shown in Figure 8b. This observation is due to large rotations of the top loading platen during the experiments. The rotation of the loading platen leads to a significant change in the overall character of the displacements applied to the specimen and, hence, a change in the global load distribution in the shell. This change results in an incremental propagation of diamond-shaped buckles around the circumference of the shell as opposed to the sudden collapse predicted for the idealized shells. The additional dynamic buckling events in the measured postbuckling response are due in part to the incremental propagation of the buckles and material failures that result in sudden stress redistribution in the shell. The material failures were attributed to interlaminar shear failures in regions of large bending gradients near the corners of the cutout. The observed and predicted results indicate the formation of a similar general instability mode.

The measured and predicted load-shortening relations for quasi-isotropic $[\mp 45 / 0 / 90]_{s}$ specimens $\mathrm{C} 4$ through $\mathrm{C} 6$ are compared in Figures 9a and 9b illustrating the effects of variations in the cutout size on the shell response. The overall trends of the predicted and measured responses are similar to those found for specimens $\mathrm{C} 1$ through $\mathrm{C} 3$. The predicted normalized buckling loads for specimens $\mathrm{C} 4, \mathrm{C} 5$, and $\mathrm{C} 6$ are 0.489 , 0.416 , and 0.386 , respectively. These values are $20.7 \%$ lower than the orthotropic buckling values on average. These results indicate that a cutout can have a greater affect on the buckling load of a quasi-isotropic shell. The experimentally measured normalized buckling loads for specimens $\mathrm{C} 4, \mathrm{C} 5$, and $\mathrm{C} 6$ are $0.466,0.433$, and 0.392 , respectively. The measured buckling loads are within $\pm 2 \%$ of the predicted buckling loads. In contrast, the predicted buckling loads for the orthotropic specimens $\mathrm{C} 1-\mathrm{C} 3$ are $19 \%$ higher on average than the measured buckling loads. These results indicate that while the buckling load of quasi-isotropic shell is more sensitive to a cutout, the magnitude of the buckling load for the orthotropic shell may be more sensitive to other unintended imperfections in the shell. While each specimen had its own unique imperfections, the overall character of the imperfections were similar.

\section{Refined Model of Specimen C1}

Results from a refined model of specimen $\mathrm{C} 1$ are presented. The results include a comparison between measured and predicted axial load versus end-shortening relations, predicted and observed deformation patterns, and predicted and measured strain response at selected locations on the shell. The values of axial load and end-shortening are normalized with respect to classical buckling values for a shell without a cutout. Due to the large problem size associated with the refined finite element model, only a portion of the compression response was predicted for cylinder $\mathrm{C} 1$. The predicted results include the prebuckling, buckling and initial postbuckling response of the specimen. 
Without the accurate modeling of the specimen imperfections and experimental loading conditions, the predicted results can be significantly different from the measured response as indicated in Figure 10a. By including the refinements discussed previously, a more accurate representation of the actual experiment can be achieved. A comparison between the predicted axial load versus end-shortening relation from a fully refined model and the measured results for specimen $\mathrm{C} 1$ is presented in Figure 10b. The superscript ' $p$ ' and superscript ' $m$ ' denote predicted and measured points, respectively. The results indicate that a dramatic improvement in the correlation between predicted response and the measured response occurs with the refined model. The predicted prebuckling response of the shell correlates well with the measured response including the nonlinear behavior of the initial prebuckling response and the effective prebuckling stiffness of the shell in the linear region of the prebuckling response. The predicted value of the normalized first local buckling load of 0.577 is approximately $0.7 \%$ greater than the measured value of 0.573 . The predicted initial postbuckling response agrees well with the measured response. However, the prediction becomes less accurate as loading continues in the postbuckling range. The reduction in accuracy is likely due in part to material failures that occur in the specimen near the cutout resulting in an overall weakening of the shell. In addition, it is possible that the linear material model and the lack of shear deformability in the element theory are no longer valid modeling assumptions in regions near the cutout due to extreme bending gradients that develop. Such modeling assumptions may result in an overly stiff response.

Predicted displacement contours and observed moire fringe patterns at selected points in the response are compared in Figures 11a-d. Figures 11a and 11b show predicted and observed postbuckling displacements associated with points $\mathrm{B}^{p}$ and $\mathrm{B}^{m}$ on the response curve in Figure 10b, respectively, and appear to agree well. The mode shape consists of two elliptical shaped buckles near the cutout aligned in a helical or skew pattern. Figures 11c and 11d compare predicted and observed secondary postbuckling mode shapes associated with points $\mathrm{D}^{p}$ and $\mathrm{D}^{m}$ in Figure $10 \mathrm{~b}$. The predicted and observed results correlate well. The local buckles near the cutout have rotated in a clock-wise sense around the cutout and additional inward buckles have formed around the circumference of the shell.

Predicted and measured strain responses from selected strain gage locations are compared in Figure 12a$d$ (refer to the inset schematic of a shell with a cutout identifying strain gage locations). Figures $12 \mathrm{a}$ and $12 \mathrm{~b}$ show the predicted and measured back-to-back far-field strain response at location \#1 and Figures $12 \mathrm{c}$ and $12 \mathrm{~d}$ show the predicted and measured strain response at location \#2 at the edge of the cutout. The results indicate that the local strain response is predicted well at these locations including the strain response near the cutout in which large strain gradients exist. The strain response presented in Figures $12 \mathrm{c}$ and $12 \mathrm{~d}$ indicate that a sudden snap-through of the top cutout edge occurs during the first local buckling event as evidenced by the strain reversal associated with segments A-B of the load-strain response curves.

It was found during the investigation that no one imperfection was more important than another in producing the increase in accuracy of the predicted results, rather, it was the combination of all measured imperfections.

\section{Concluding Remarks}

Result from a numerical and experimental study of the response of compression-loaded composite shells with rectangular cutouts are presented. The response of shells with quasi-isotropic and orthotropic in-plane stiffness properties and three cutout sizes are discussed. Results indicate that a nonlinear interaction between out-of-plane deformations and in-plane stresses near a cutout in a compression-loaded shell causes a local buckling response to occur near the cutout. Additional load can be applied to the shell in the postbuckling range before collapsing into the general instability mode. Each buckling event is accompanied by a reduction in the effective axial stiffness due to the formation of large deformations in the shell. The compression response is strongly influenced by the local displacements and internal load distribution near the cutout. These local displacements and internal load distributions are affected by the size of the cutout, material properties, and imperfections in the shell.

Results indicate that an increase in the size of the cutout causes a reduction in the buckling load of the shell and the magnitude of the load reduction associated with the buckling response. Numerical results predict that the buckling load of a compression-loaded axially stiff orthotropic shell is less sensitive to the addition of a cutout than the buckling load of a quasiisotropic shell. The experimental results seem to indicate that the buckling load of an orthotropic shell with a cutout may be more sensitive to other imperfections in the shell than the buckling load of a quasi-isotropic shell with an identical cutout.

Results from a refined model of a selected cylinder specimen, including measured initial geometric imperfections, nonuniform load introduction, shell wall thickness variations and material property variations, correlate well with measured and observed results. The 
results are important for establishing credibility in the predictive capabilities of the analysis methods employed. The results indicate that accurate models are possible for structures with complex nonlinear behavior under static and dynamic loading conditions.

\section{Acknowledgments}

This work was supported by the NASA Graduate Student Researchers Program and NASA Langley Research Center, Grant Number NGT-57256.

\section{References}

${ }^{1}$ Lur'e, A. I., "Statics of Thin-walled Elastic Shells," State Publishing House of Technical and Theoretical Literature, Moscow, 1947; translation, AEC-tr-3798, Atomic Energy Commission, 1959.

${ }^{2}$ Lekerkerker, J. G., "On the Stress Distribution in Cylindrical Shells Weakened by a Circular Hole," Ph.D. dissertation, Technological University, Delft, The Netherlands, 1965.

${ }^{3}$ Brogan, F. A. and Almroth, B. O., "Buckling of Cylinders with Cutouts," AIAA Journal, Vol. 8, No. 2, February 1970, pp. 236-240.

${ }^{4}$ Tennyson, R. C., "The Effects of Unreinforced Circular Cutouts on the Buckling of Circular Cylindrical Shells," Journal of Engineering for Industry, Transactions of the American Society of Mechanical Engineers, Vol. 90, November 1968, pp. 541-546.

${ }^{5}$ Starnes, J. H., "The Effect of a Circular Hole on the Buckling of Cylindrical Shells," Ph. D. dissertation, California Institute of Technology, Pasadena, California, 1970.

${ }^{6}$ Hilburger, M. W., "Numerical And Experimental Study of the Compression Response of Composite Cylindrical Shells with Cutouts," Ph. D. dissertation, University of Michigan, Ann Arbor, Michigan, 1998.

${ }^{7}$ Brogan, F. A., Rankin, C. C., and Cabiness, H. D., "STAGS User Manual," Lockheed Palo Alto Research Laboratory, Report LMSC P032594, 1994.

${ }^{8}$ Riks, E., "The Application of Newton's Method to the Problem of Elastic Stability," ASME Journal of Applied Mechanics, Vol. 39, 1972, pp. 1060-1066.

${ }^{9}$ Riks, E., and Rankin, C. C., "Computer Simulation of Dynamic Buckling Phenomena Under Quasi-static Loads," presented, Euromech Colloquium 317, March 1994, Liverpool, UK.
Table 1: AS4/3502 Material Properties

\begin{tabular}{cccc}
\hline \hline$E_{1}, \mathrm{Msi}$ & $E_{2}, \mathrm{Msi}$ & $G_{12}, \mathrm{Msi}$ & $\nu_{12}$ \\
\hline 21.5 & 1.61 & 0.93 & 0.295 \\
\hline \hline
\end{tabular}

Table 2: Summary of Cylinder Code, Layup, and Cutout Dimensions

\begin{tabular}{|c|c|c|}
\hline $\begin{array}{c}\text { Cylinder } \\
\text { Identification } \\
\text { Code }\end{array}$ & $\begin{array}{c}\text { Wall } \\
\text { Construction }\end{array}$ & $\begin{array}{c}a, b \\
\text { in., in. }\end{array}$ \\
\hline C1 & & $1.0,1.0$ \\
C2 & {$\left[ \pm 45 / 0_{2}\right]_{s}$} & $2.0,1.0$ \\
C3 & & $1.0,2.0$ \\
\hline C4 & & $1.0,1.0$ \\
C5 & {$[ \pm 45 / 0 / 90]_{s}$} & $2.0,1.0$ \\
C6 & & $1.0,2.0$ \\
\hline \hline
\end{tabular}

Table 3: Average Adjusted Cylinder Geometry for C1

\begin{tabular}{ccc}
\hline \hline$R$, in. & $L$, in. & $t$, in. \\
\hline 7.932 & 16.008 & 0.0379 \\
\hline \hline
\end{tabular}

Table 4: Average Thickness Adjusted AS4/3502 Material Properties

\begin{tabular}{cccc}
\hline \hline$E_{1}, \mathrm{Msi}$ & $E_{2}, \mathrm{Msi}$ & $G_{12}, \mathrm{Msi}$ & $\nu_{12}$ \\
\hline 22.9 & 1.73 & 1.01 & 0.291 \\
\hline \hline
\end{tabular}

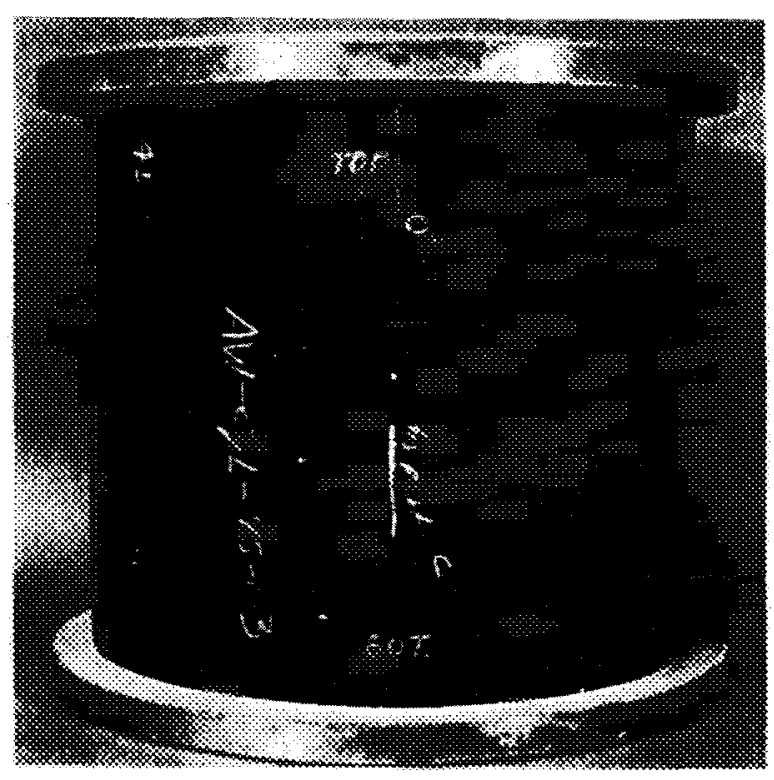

Figure 1: Typical shell specimen. 

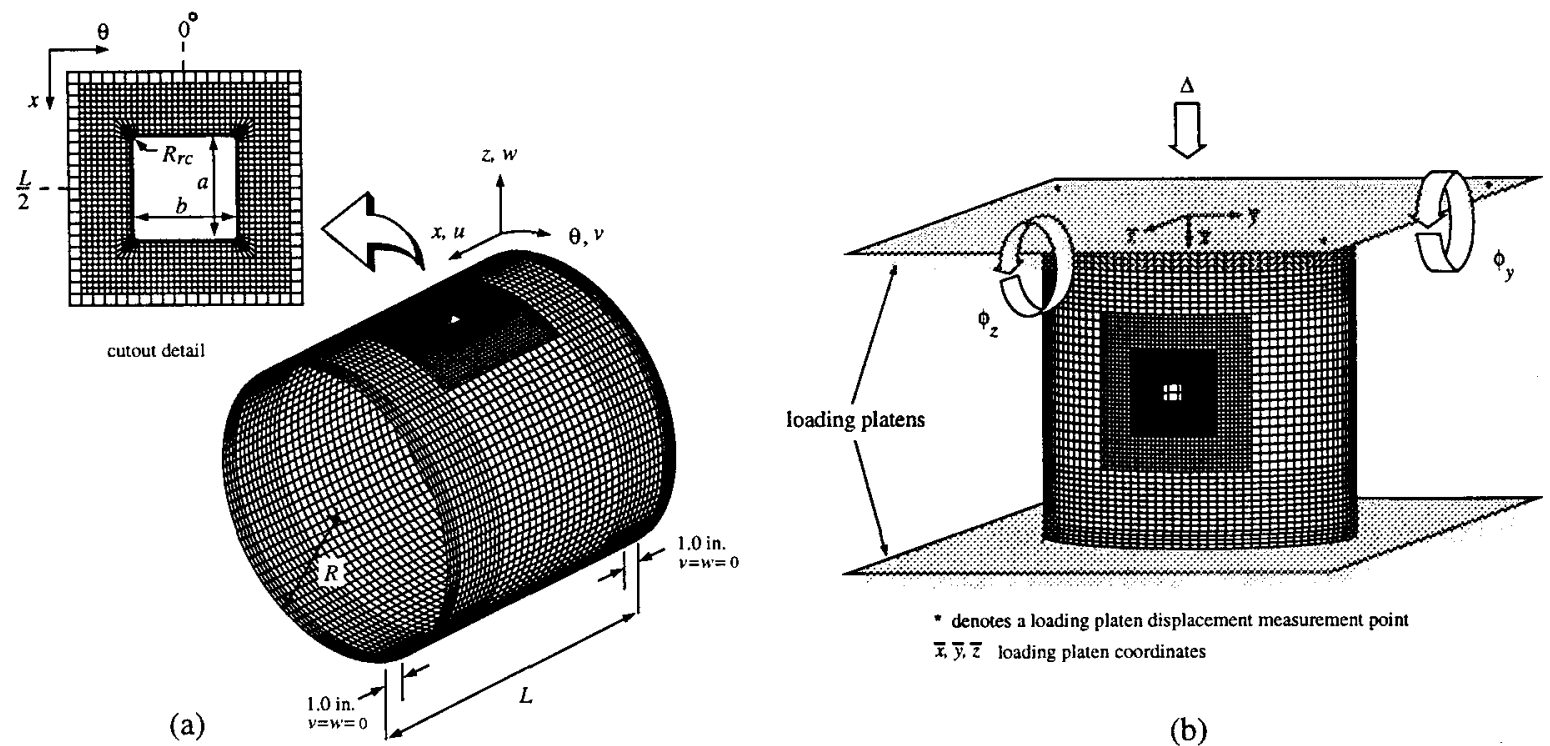

Figure 2: Shell geometry and boundary conditions.

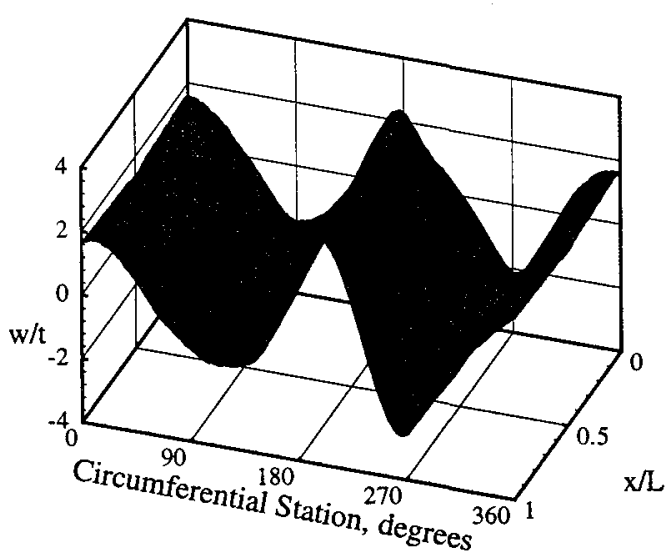

(a) Normalized geometric imperfections

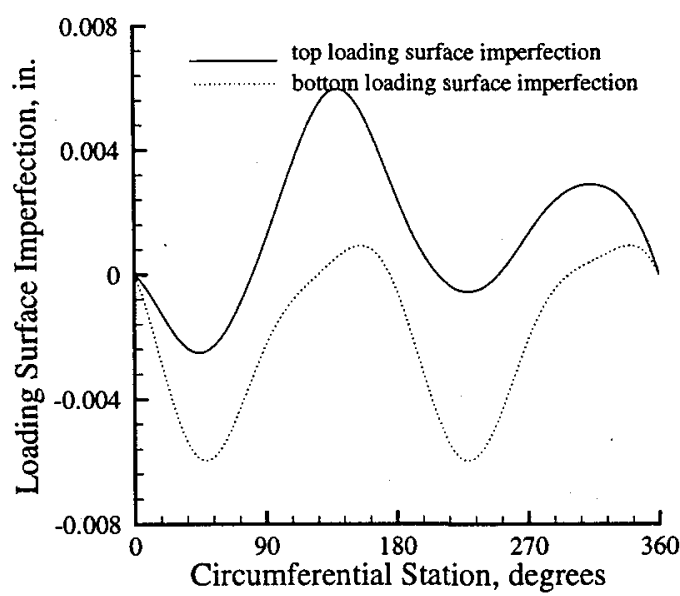

(c) Loading surface profiles

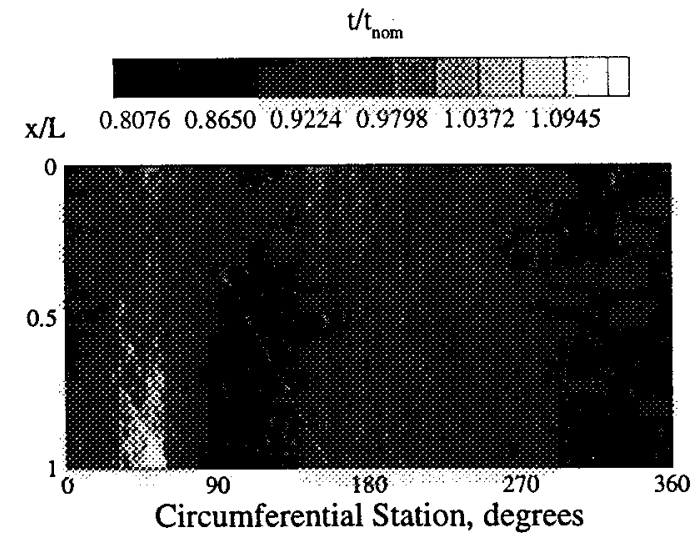

(b) Normalized wall thickness distribution

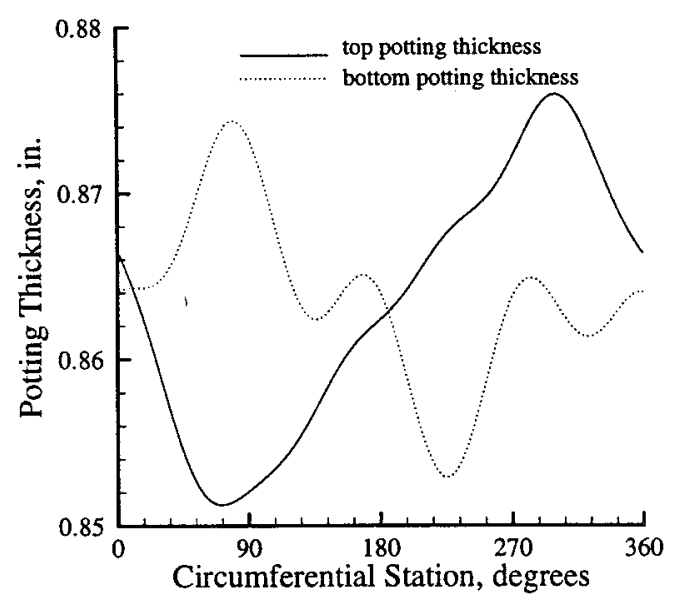

(d) Potting thickness distribution

Figure 3: Measured imperfections for specimen $\mathrm{C} 1$. 


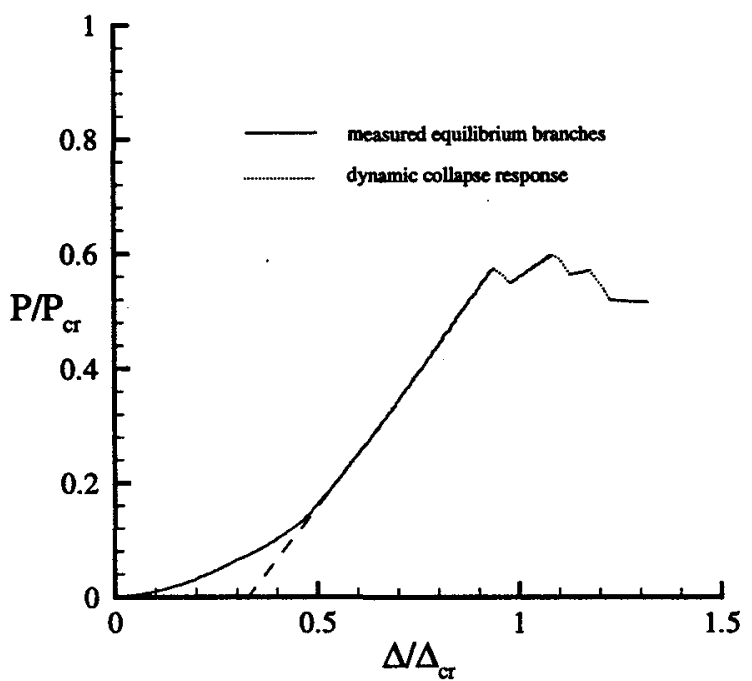

(a)

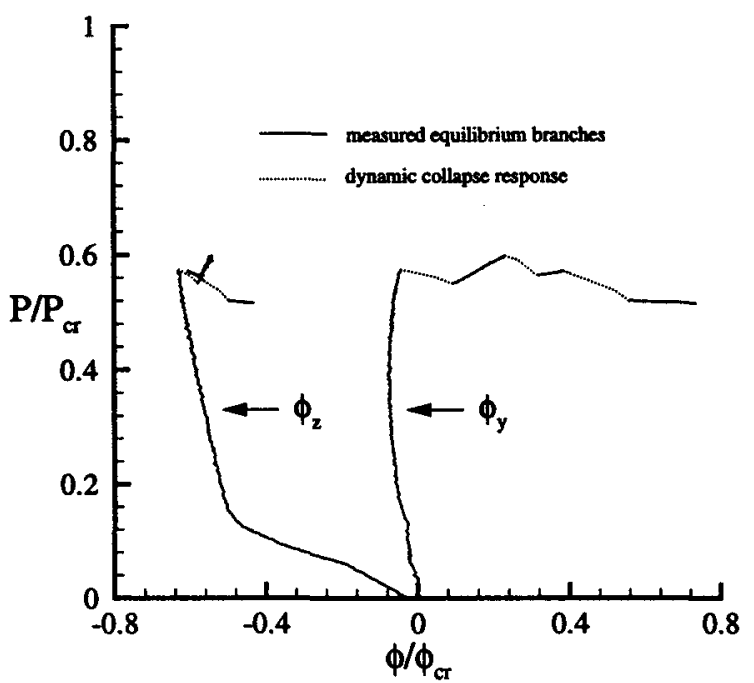

(a)

Figure 4: (a) Typical measured load-shortening relation. (b) Typical measured load-rotation relations.

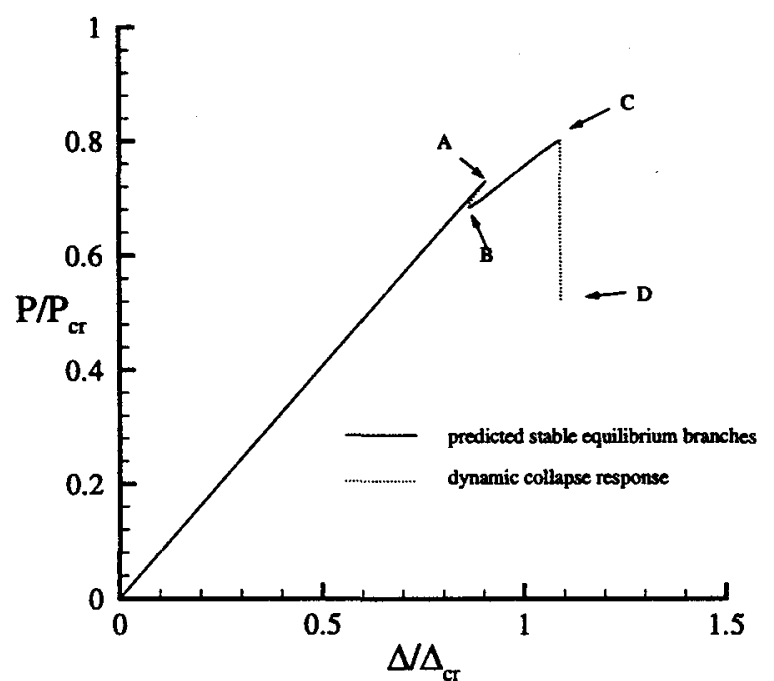

(a)

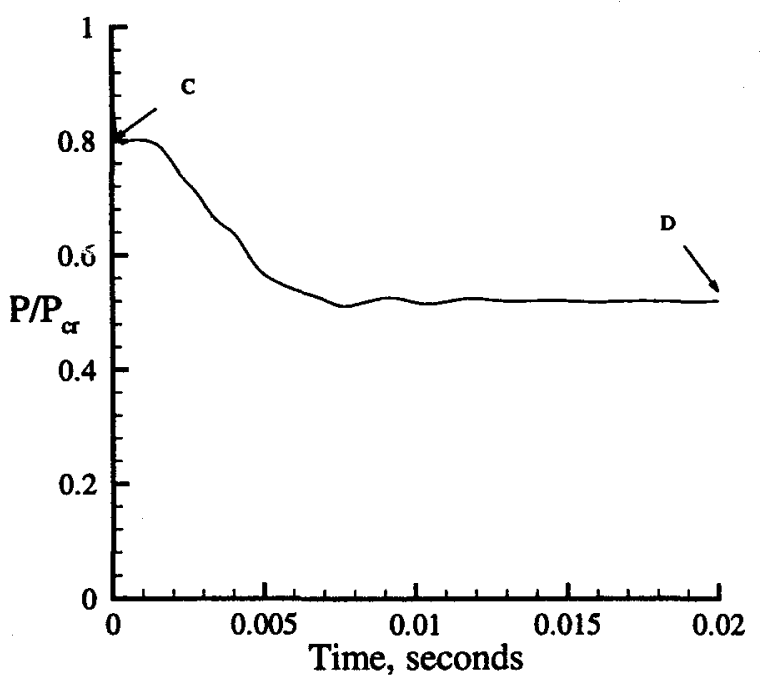

(a)

Figure 5: (a) Predicted load-shortening relation for specimen C1, geometrically perfect model. (b) Predicted load time history for the collapse response represented by segment C-D in Figure 5a. 


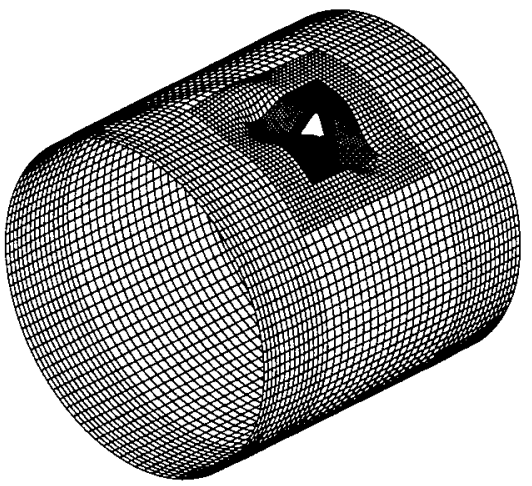

(a)

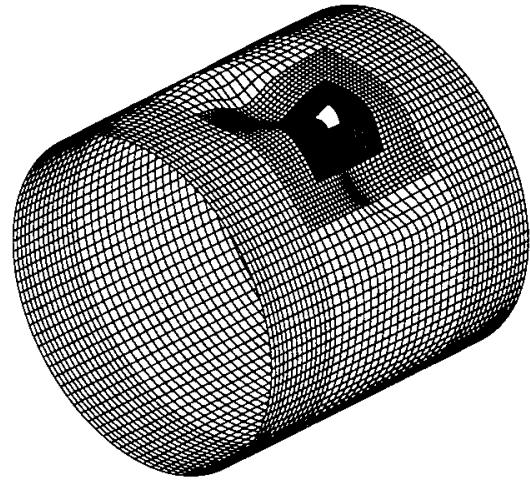

(b)

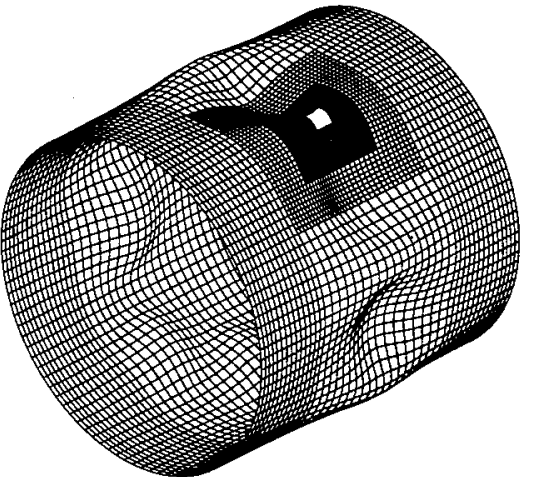

(c)

Figure 6: Deformation patterns associated with selected points on the load-shortening response curve in Figure 5a; (a) point $\mathrm{B}$, (b) point $\mathrm{C}$, (c) point $\mathrm{D}$.

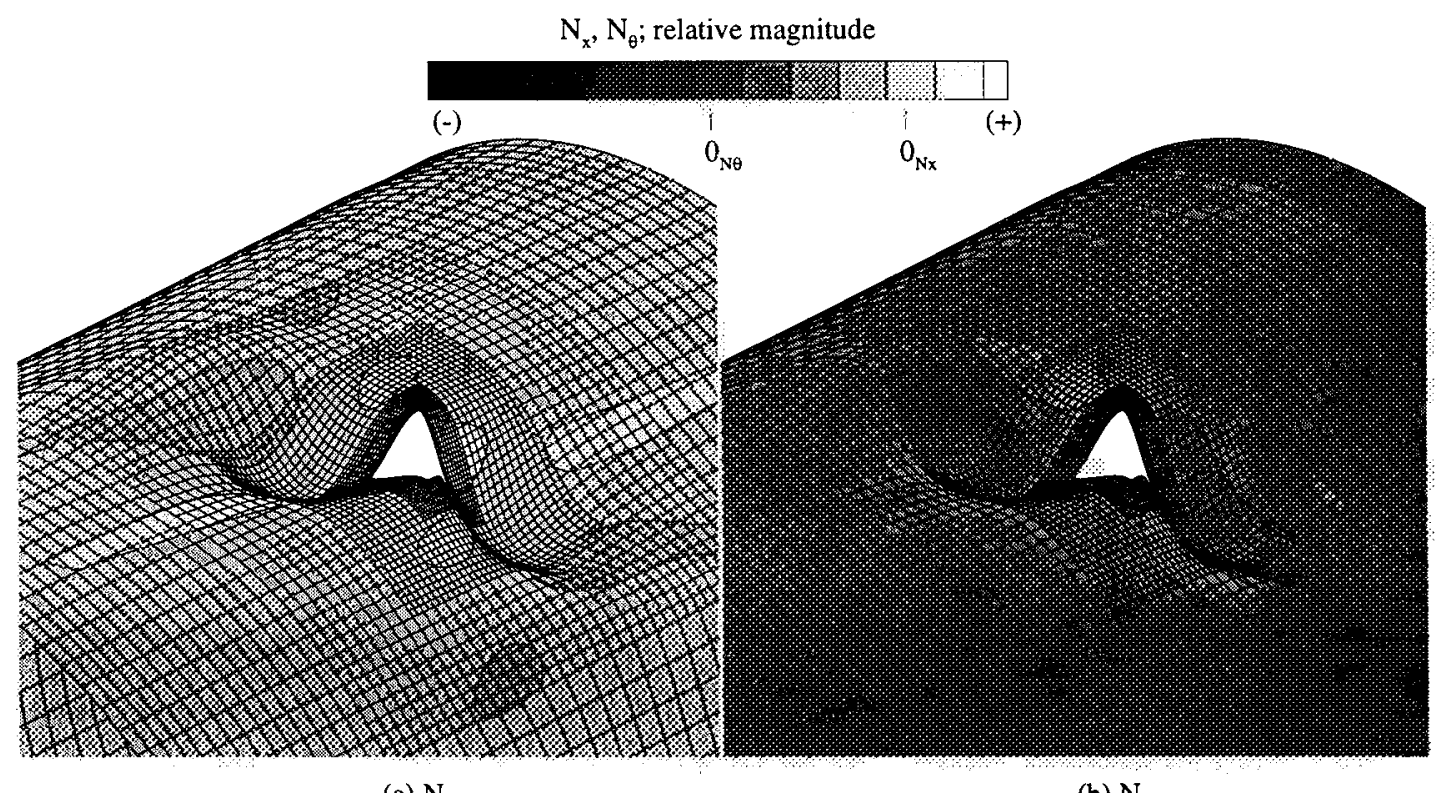

(a) $\mathrm{N}_{\mathrm{x}}$

(b) $\mathrm{N}_{\theta}$

Figure 7: Buckling in-plane stress contours for geometrically perfect model of specimen $\mathrm{C} 1$ associated with point $B$ on the load-shortening response curve in Figure 5a (magnified view). 


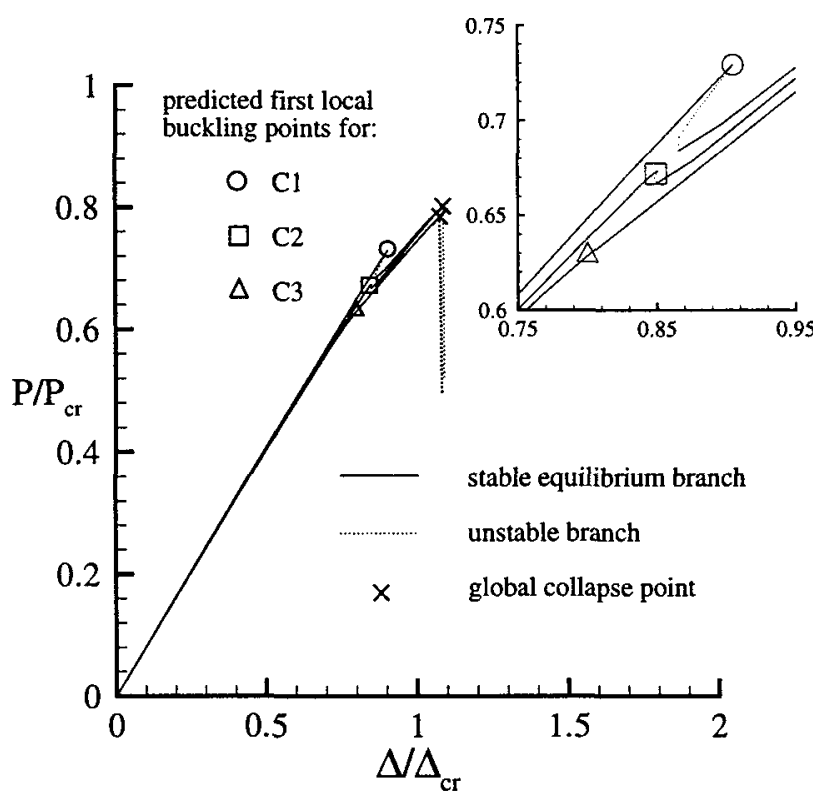

(a)

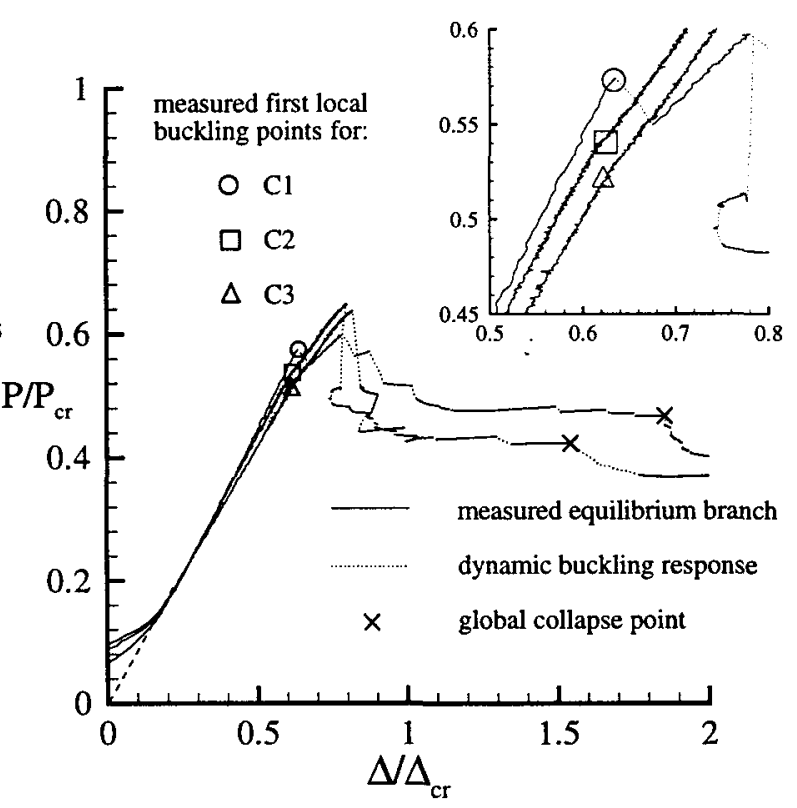

(b)

Figure 8: Comparison between predicted and measured load-shortening relations for orthotropic specimens with cutouts, C1-C3, (a) predicted load-shortening response of geometrically prefect shells, (b) measured loadshortening response.

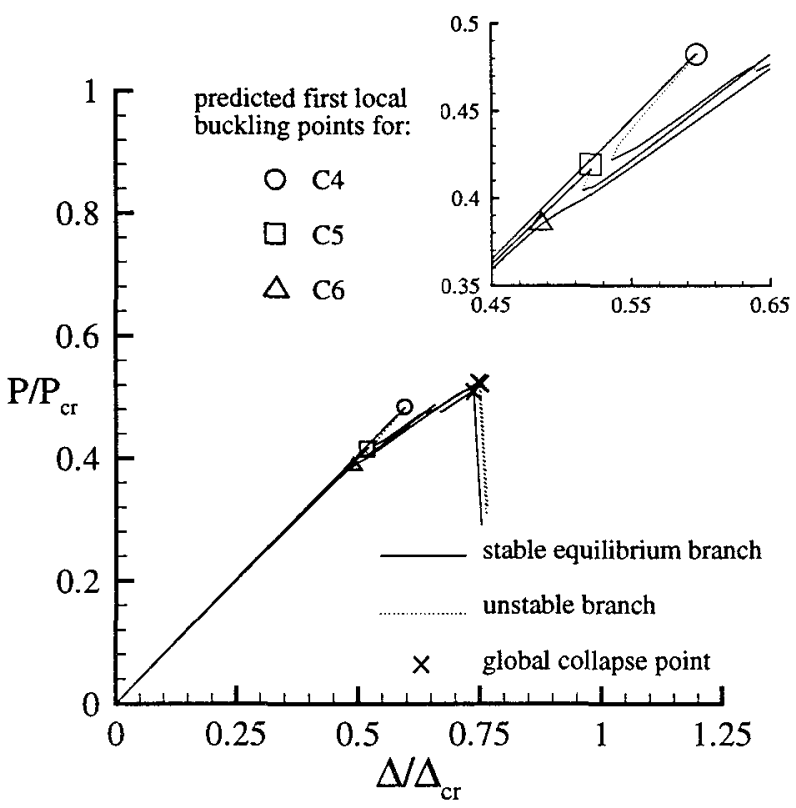

(a)

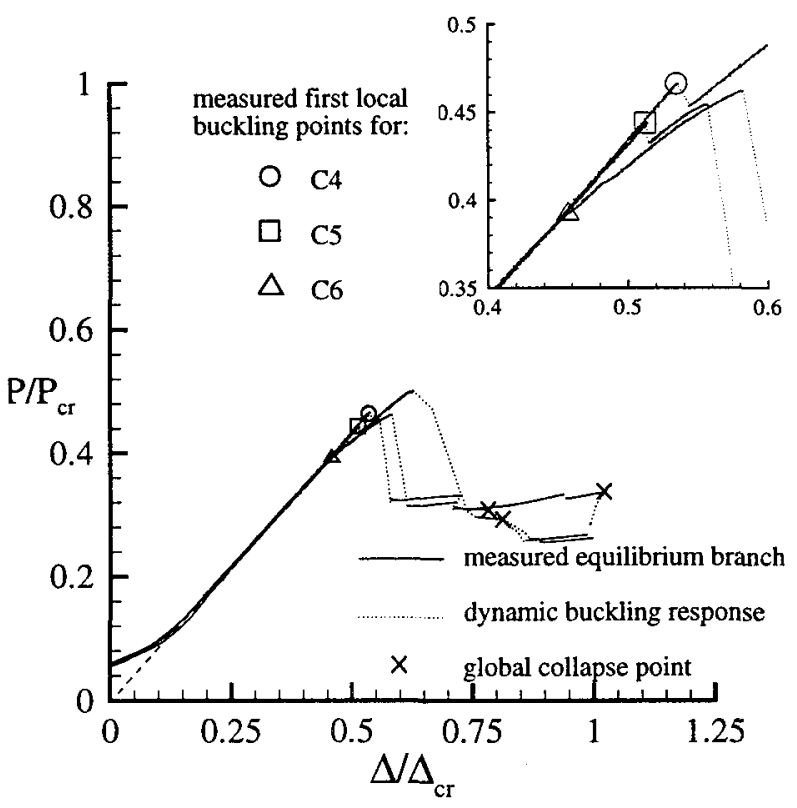

(b)

Figure 9: Comparison between predicted and measured load-shortening relations for quasi-isotropic specimens with cutouts, C4-C6, (a) predicted load-shortening response of geometrically prefect shells, (b) measured loadshortening response. 


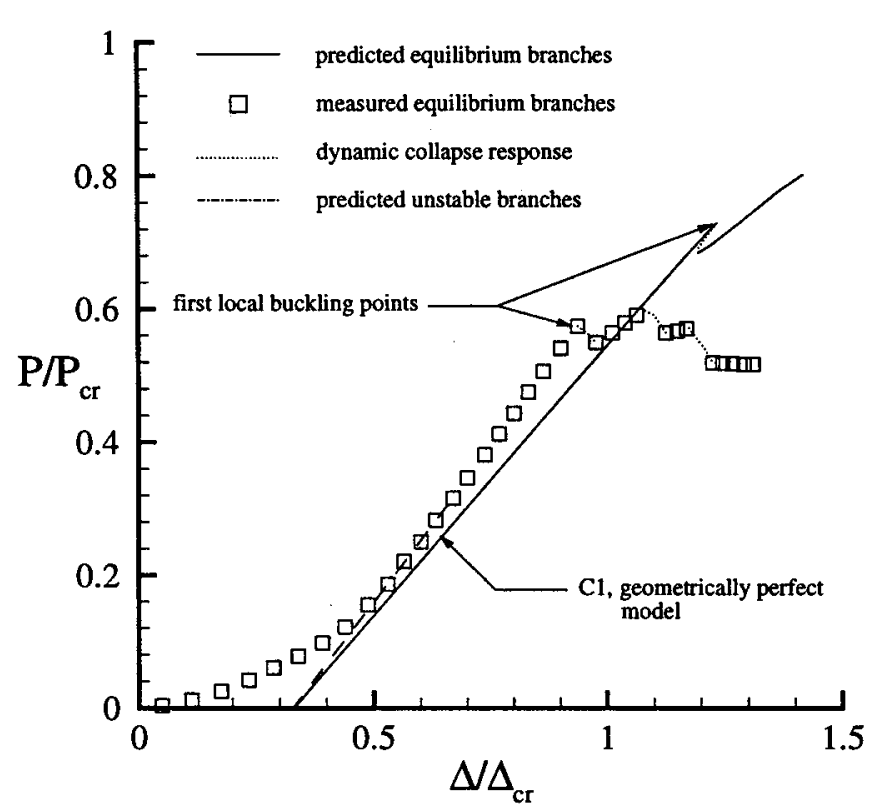

(a)

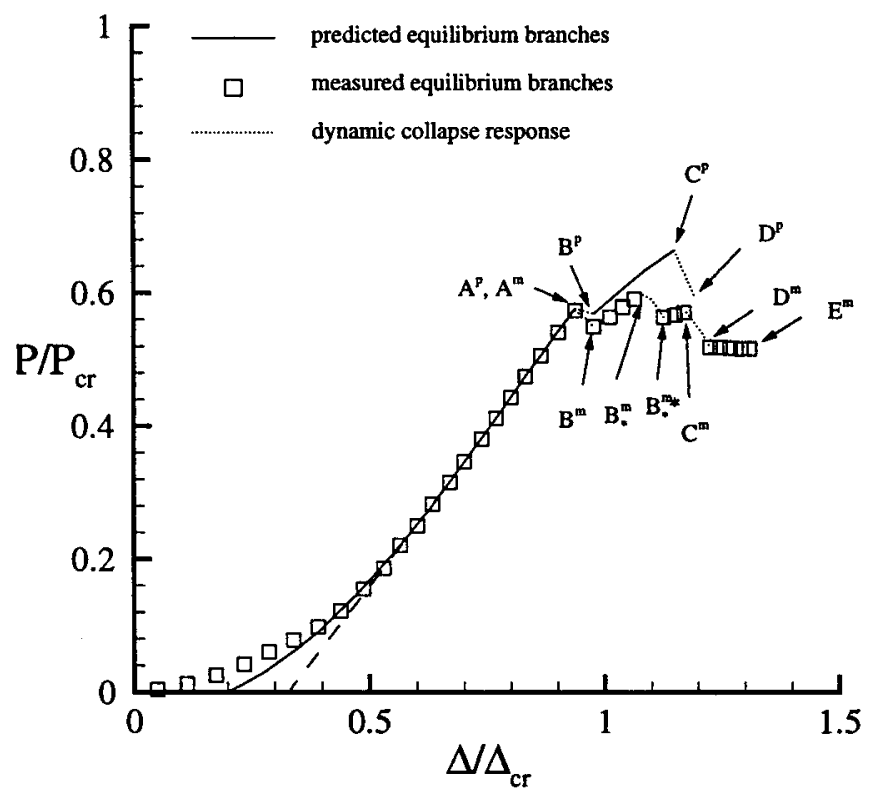

(b)

Figure 10: Comparison between predicted and measured load-shortening response for specimen $\mathrm{C} 1$, (a) perfect model prediction versus measured results, (b) refined model prediction versus measured results. 



Figure 11: Comparison between predicted and measured shell displacements for specimen $\mathrm{C} 1$ at selected points in the response, (a) predicted displacement contours associated with point $\mathrm{B}^{p}$ in Figure $10 \mathrm{~b},(\mathrm{~b})$ observed moirefringe patterns associated with point $\mathrm{B}^{m}$ in Figure $10 \mathrm{~b},(\mathrm{c})$ predicted displacement contours associated with point $\mathrm{D}^{p}$ in Figure 10b, (d) observed moire-fringe patterns associated with point $\mathrm{D}^{m}$ in Figure 10b. 


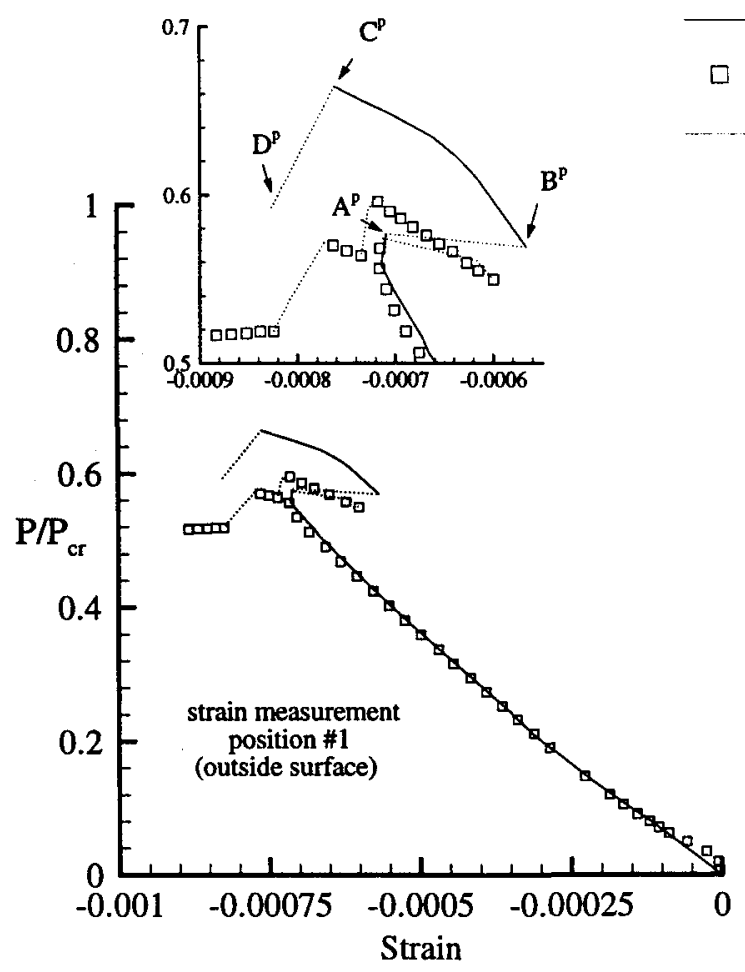

predicted strain response

measured strain response

dynamic buckling response
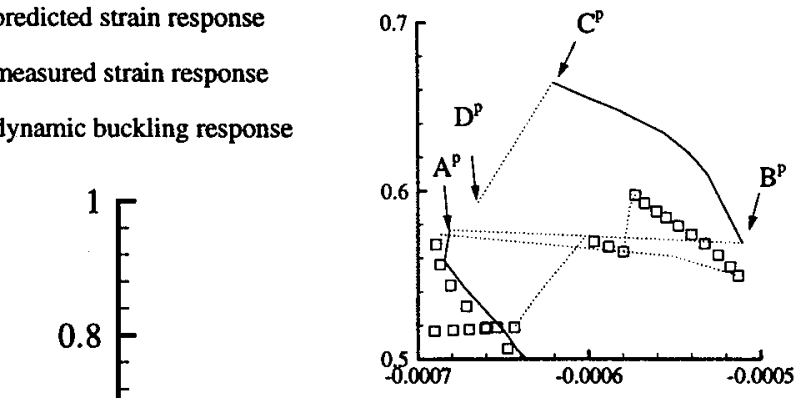

(a)

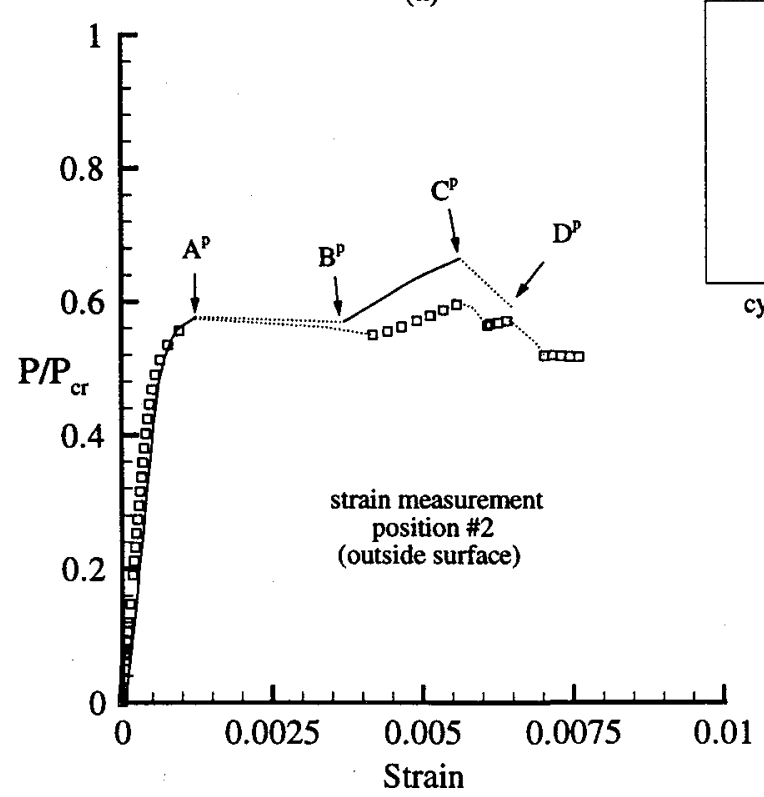

(c)

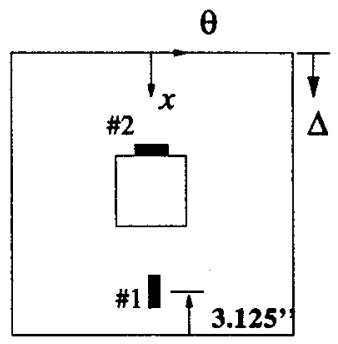

cylinder schematic

$\mathrm{P} / \mathrm{P}$

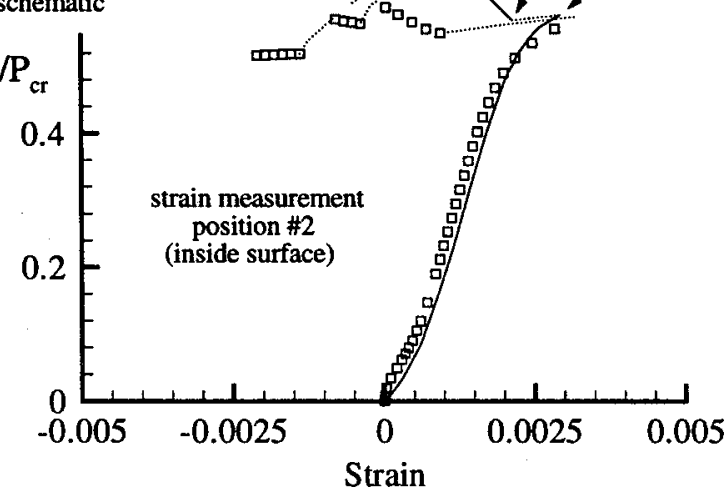

(d)

Figure 12: Comparison between predicted and measured strains at selected locations on specimen C1, (a) predicted and measured strains from gage position \#1 (outer surface), (b) predicted and measured strains from gage position \#1 (inner surface), (c) predicted and measured strains from gage position \#2 (outer surface), (c) predicted and measured strains from gage position \#2 (inner surface) 